\title{
Adverbs in a Modern Type Theory
}

\author{
Stergios Chatzikyriakidis \\ Dept of Computer Science, Royal Holloway, Univ of London \\ Egham, Surrey TW20 0EX, U.K; Open University of Cyprus \\ stergios.chatzikyriakidis@cs.rhul.ac.uk
}

\begin{abstract}
This paper is the first attempt to deal with aspects of the semantics of adverbs within a modern type theoretical setting. A number of issues pertaining to the semantics of different classes of adverbs like verididality and intensionality will be discussed and further shown to be captured straightforwardly within a modern type theoretical setting. In particular, I look at the issue of veridicality and show that the inferences associated with veridical adverbs can be dealt with via typing alone, i.e. without the aid of meaning postulates. In case of intensional adverbs like intentionally or allegedly, I show that these can be captured by making use of the type theoretical notion of context, i.e. without the use of possible worlds.
\end{abstract}

\section{Introduction}

The linguistic class of adverbs is a well-known example where members of a single category exhibit a vast non-homogeneity as regards their semantic interpretation. A number of different semantic classes can be distinguished for adverbs according to the semantics they give rise to. However, it seems that an abstraction to a tripartite classification can be made possible in the sense of [21]. According to this classification, there are three basic classes of adverbials with further subclassifications for each class: a) predicational, b) participant oriented and c) functional adverbials. ${ }^{1}$ Note that this classification is for adverbials in general, and not adverbs only. In this paper, we concentrate on a subset of predicational adverbs that constitute the main bulk of adverbs. Predicational adverbs comprise a class of adverbs that are further subcategorized into: a) sentence adverbs and b) verb-related adverbs. In the former category, we further find: a) subject oriented adverbs like arrogantly, b) speaker-oriented adverbs like honestly, intentionally and surprisingly, and c) domain adverbs like botanically and mathematically. Verb related adverbs on the other hand include: a) mental attitude adverbs like reluctantly, b) manner adverbs like skilfully and slowly, and c) degree adverbs like deeply. ${ }^{2}$

\footnotetext{
${ }^{1}$ There are also syntactic criteria that are used in the literature to distinguish between the types of adverbs. We do not discuss syntax in this paper, and as such, no such criteria are discussed. See $[11,6,8,9]$ among many others for discussions of syntactic relevance.

${ }^{2}$ See [21] for more details on the classification.
} 
There are various proposals pertaining to the semantics of adverbs. However, most of the approaches boil down to two major ways of looking at adverbs. The first of these is the operator approach proposed within the Montagovian tradition, while the latter concerns approaches within the (neo)-Davidsonian event-related tradition. Both lines of approach have their merits as well as their disadvantages. According to the operator approach (see $[24,28,12]$ among others), adverbs are seen as functors which return the same type as that of their argument, further distinguishing between adverbs that take a truth value (or a proposition) to return a truth value (or proposition) and adverbs that take a set (or concept) to return a set (or concept): ${ }^{3}$

(1) Extensional: $(e \rightarrow t) \rightarrow(e \rightarrow t)$

Intensional: $(s \rightarrow(e \rightarrow t)) \rightarrow(s \rightarrow(e \rightarrow t))$

(2) Extensional: $(t \rightarrow t)$

Intensional: $(s \rightarrow t) \rightarrow(s \rightarrow t)$

The typings above correspond to the distinction between sentence type adverbs (e.g. evaluative adverbs like fortunately) and VP-adverbs (e.g. manner adverbs), while the intensionalized versions of the typings make the rather welcoming prediction that in VP-adverbs, opaque contexts should arise for the object but not for the subject, a prediction which is borne out from the facts (see $[12,28]$ and $[21]$ for a summary). ${ }^{4}$

On the other hand, approaches within the tradition initiated by Davidson [7], argue for an analysis where adverbs can be seen as providing restrictions w.r.t the event denoted by the sentence/proposition in each case. In effect, adverbs in these approaches are assumed to modify the event in some way. According to Davidson, each sentence involves an implicit event argument. This event argument is then assumed to be modified by adverbs and adverbials forming a simple conjunction in a first-order logic language. For example, a sentence like John walks slowly will receive the following semantics in a Davidsonian setting: ${ }^{5}$

(3) $\exists e: W A L K I N G(e) \wedge A G E N T(e, J o h n) \wedge S L O W(e)$

In this paper, I present a first account of some aspects og the semantics of adverbs based on modern type theories, i.e. type theories within the tradition of Martin-Löf. In particular, an expressive and computationally attractive language, that of UTT with coercive subtyping $[16,15,18]$ will be used in order to present an account of various aspects of adverbial modification that, as it will

\footnotetext{
${ }^{3}$ Within the simple type theory used in Montague Grammar, $e$ is the type of individuals, $t$ is the type of truth-values and $s$ the type of world-time pairs.

${ }^{4}$ An example in case would involve a VP-adverb like intentionally in a sentence like Oedipus intentionally married Jocaste. Under the intensionalized VP-adverb typing, it does not follow that Oedipus intentionally married his mother. See the analysis and discussion in $\S(3.2)$.

${ }^{5} e$ stands for event here, not to be conflated with the type $e$ of individuals used in MG.
} 
be argued, fares well with respect to a number of well-known semantic issues associated with adverbial modification.

\section{MTT semantics: a brief intro}

A Modern Type Theory (MTT) is a variant of a class of type theories in the tradition initiated by the work of Martin-Löf [22,23], which have dependent and inductive types, among others. We choose to call them Modern Type Theories in order to distinguish them from Church's simple type theory [5] that is commonly employed within the Montagovian tradition in formal semantics.

Among the variants of MTTs, we are going to employ the Unified Theory of dependent Types (UTT) [13] with the addition of the coercive subtyping mechanism (see, for example, $[14,19]$ and below). UTT is an impredicative type theory in which a type Prop of all logical propositions exists. ${ }^{6}$ This stands as part of the study of linguistic semantics using MTTs rather than simply typed ones. These issues have already been discussed in $[16,17,1,2,4]$ among others. In what follows I provide a brief discussion on some of the features of MTTs relevant to the paper.

\subsection{Type many-sortedness and CNs as types}

In Montague Grammar (MG, [25]), the underlying logic (Church's simple type theory [5]) can be seen as 'single-sorted' in the sense that there is only one type $e$ of all entities. The other types such as $t$ of truth values and the function types generated from $e$ and $t$ do not stand for types of entities. Thus, there are no finegrained distinctions between the elements of type $e$ and as such all individuals are interpreted using the same type. For example, John and Mary have the same type in simple type theories, the type $e$ of individuals. An MTT, on the other hand, can be regarded as a 'many-sorted' logical system in that it contains many types and as such one can make fine-grained distinctions between individuals and further use those different types to interpret subclasses of individuals. For

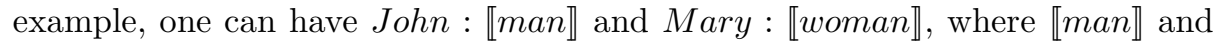
$\llbracket w o m a n \rrbracket$ are different types.

An important trait of MTT-based semantics is the interpretation of common nouns (CNs) as types [27] rather than sets or predicates (i.e., objects of type $e \rightarrow t)$ as it is the case within the Montagovian tradition. The CNs man, human,

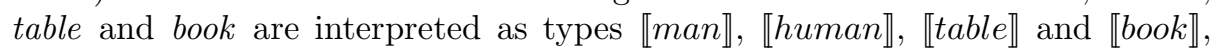
respectively. Then, individuals are interpreted as being of one of the types used to interpret CNs. The interpretation of CNs as Types is also a prerequisite in order for the subtyping mechanism to work. This is because, assuming CNs to be predicates, subtyping would go wrong given contravariance of function types. ${ }^{7}$

\footnotetext{
6 This is similar to simple type theory where a type $t$ of truth values exists.

${ }^{7}$ See [3] for more information. See also [17] for further philosophical argumentation on the choosing to represent $\mathrm{CNs}$ as types. Furthermore, one anonymous reviewer
} 


\subsection{Dependent typing and universes}

One of the basic features of MTTs is the use of Dependent Types. A dependent type is a family of types depending on some values. Here two basic constructors for dependent types are explained, $\Sigma$ and $\Pi$, both highly relevant for the study of linguistic semantics.

The constructor/operator $\Sigma$ is a generalization of the Cartesian product of two sets that allows the second set to depend on values of the first. For

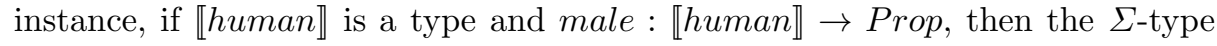
$\Sigma h: \llbracket$ human』. male $(h)$ is intuitively the type of humans who are male.

More formally, if $A$ is a type and $B$ is an $A$-indexed family of types, then $\Sigma(A, B)$, or sometimes written as $\Sigma x: A . B(x)$, is a type, consisting of pairs $(a, b)$ such that $a$ is of type $A$ and $b$ is of type $B(a)$. When $B(x)$ is a constant type (i.e., always the same type no matter what $x$ is), the $\Sigma$-type degenerates into product type $A \times B$ of non-dependent pairs. $\Sigma$-types (and product types) are associated projection operations $\pi_{1}$ and $\pi_{2}$ so that $\pi_{1}(a, b)=a$ and $\pi_{2}(a, b)=b$, for every $(a, b)$ of type $\Sigma(A, B)$ or $A \times B$.

The linguistic relevance of $\Sigma$-types can be directly appreciated once we understand that in its dependent case, $\Sigma$-types can be used to interpret linguistic phenomena of central importance, like for example adjectival modification [27]. For example, handsome man is interpreted as a $\Sigma$-type (4), the type of handsome men (or more precisely, of those men together with proofs that they are handsome): ${ }^{8}$

(4) $\Sigma m: \llbracket m a n \rrbracket \cdot \llbracket h a n d s o m e \rrbracket(m)$

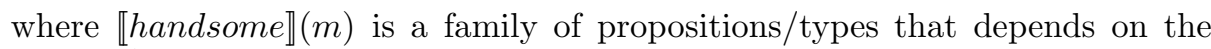
man $m .^{9}$

The other basic constructor for dependent types is $\Pi$. $\Pi$-types can be seen as a generalization of the normal function space where the second type is a

asks about any potential drawbacks of assuming CNs to be types. He mentions cases of noun compounding, asking how such cases would be treated. As far as I am concerned, these cases have not been dealt with yet under such an approach. Thus, I would be reluctant to answer such a question. The idea of defining type constructors that correspond to noun constructors seems viable, but the exact details of these constructors, given the compositionality issues abound in noun compounding, are unknown to me. On a more general note, it seems to me that noun compounding is a problematic issue for any formal semantic theory and not only for the approach pursued here. It is thus undeniable that a formal semantic theory should be able to address these cases. However, for the moment such an account does not exist within the framework discussed in this paper. I leave this issue as a subject of future research.

${ }^{8}$ It should be kept in mind that every proposition P:Prop is a type and that an object of a proposition is called a proof.

${ }^{9}$ Adjectival modification is a notoriously difficult issue and as such not all cases of adjectives can be captured using a $\Sigma$ type analysis. For a proper treatment of adjectival modification within this framework, see [2]. 
family of types that might be dependent on the values of the first. A $\Pi$-type $\Pi(A, B)$ degenerates to the function type $A \rightarrow B$ in the non-dependent case. In more detail, when $A$ is a type and $P$ is a predicate over $A, \Pi x: A . P(x)$ is the dependent function type that, in the embedded logic, stands for the universally quantified proposition $\forall x: A . P(x) .{ }^{10}$ For example, the following sentence (5) is interpreted as (6):

(5) Every man walks.

(6) $\Pi x: \llbracket m a n \rrbracket \cdot \llbracket w a l k \rrbracket(x)$

Type Universes. An advanced feature of MTTs, which will be shown to be very relevant in interpreting NL semantics, is that of universes. Informally, a universe is a collection of (the names of) types put into a type [23]. ${ }^{11}$ For example, one may want to collect all the names of the types that interpret common nouns into a universe CN : Type. The idea is that for each type $A$ that interprets a common noun, there is a name $\bar{A}$ in $\mathrm{CN}$. For example,

$$
\overline{\llbracket m a n \rrbracket}: \mathrm{CN} \quad \text { and } \quad T_{\mathrm{CN}}(\overline{\llbracket m a n \rrbracket})=\llbracket \operatorname{man} \rrbracket .
$$

In practice, we do not distinguish a type in $\mathrm{CN}$ and its name by omitting the overlines and the operator $T_{\mathrm{CN}}$ by simply writing, for instance, $\llbracket m a n \rrbracket: \mathrm{CN}$. Thus, the universe CNincludes the collection of the names that interpret common nouns. For example, in $\mathrm{CN}$, we shall find the following types:

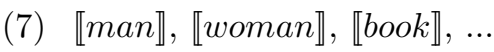

(8) $\Sigma m: \llbracket m a n \rrbracket \cdot \llbracket h a n d s o m e \rrbracket(m)$

(9) $G_{R}+G_{F}$

where the $\Sigma$-type in ( 8 is the proposed interpretation of 'handsome man' and the disjoint sum type in (9) is that of 'gun' (the sum of real guns and fake guns). ${ }^{12}$ Interesting applications of the use of universes can be proposed. For example, we can propose types for quantifiers and VP adverbs that extend over the universe $\mathrm{CN}[20]$ as well as a type for coordination extending over the universe of all linguistic types LType [1]. We will see the relevance of universes further on in this paper, when VP-adverbs are going to be discussed.

${ }^{10}$ The meticulous reader will notice that I make use of both $\Pi$ and $\forall$ in this paper. Formally $\forall$ is just a notation for $\Pi$. When $\Pi x$ :A.P:Prop we write $\forall x$ :A.P instead.

11 There is quite a long discussion on how these universes should be like. In particular, the debate is largely concentrated on whether a universe should be predicative or impredicative. A strongly impredicative universe $U$ of all types (with $U: U$ and $\Pi$ types) is shown to be paradoxical [10] and as such logically inconsistent. The theory UTT used here has only one impredicative universe Prop (representing the world of logical formulas) together with infinitely many predicative universes which as such avoids Girard's paradox (see [13] for more details).

12 The use of disjoint sum types was proposed by [2] in order to deal with privative modification. The interested reader is directed there for details. 


\subsection{Subtyping}

Coercive subtyping $[14,19]$ provides an adequate framework to be employed for MTT-based formal semantics $[15,18] .{ }^{13}$ It can be seen as an abbreviation mechanism: $A$ is a (proper) subtype of $B(A<B)$ if there is a unique implicit coercion $c$ from type $A$ to type $B$ and, if so, an object $a$ of type $A$ can be used in any context $\mathfrak{C}_{B}[-]$ that expects an object of type $B: \mathfrak{C}_{B}[a]$ is legal (well-typed) and equal to $\mathfrak{C}_{B}[c(a)]$.

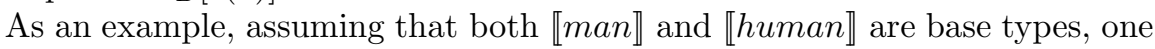
may introduce the following as a basic subtyping relation:

$(10) \llbracket m a n \rrbracket<\llbracket h u m a n \rrbracket$

In case that $\llbracket m a n \rrbracket$ is defined as a composite $\Sigma$-type (see $\S 2.2$ below for details),

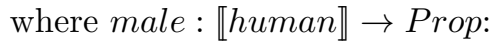

$(11) \llbracket m a n \rrbracket=\Sigma h: \llbracket h u m a n \rrbracket \cdot \operatorname{male}(h)$

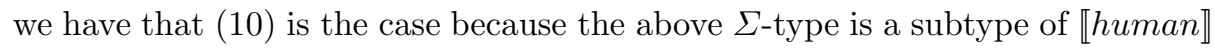
via the first projection $\pi_{1}$ :

$(12)(\Sigma h: \llbracket h u m a n \rrbracket$. male $(h))<_{\pi_{1}} \llbracket$ human

\section{Adverbs in MTTs}

A proper treatment of adverbs is lacking in the literature on MTTs. The classic work on MTT semantics, i.e. [27] only discusses time adverbials, and these not per se, but in order to deal with tense. No explicit discussion on either the typing or the semantics of adverbs is done. The first discussion, though in passing, of the correspondence of the typings of sentence and VP level adverbs in MTTs was proposed by Luo [16], followed by work on inference by $[3,4]$. In the latter two papers, adverbs are discussed in order to deal with quantifier inference cases involving adverbs like the one shown below:

(13) Some delegates finished the survey on time.

Did any delegate finish the survey? [Yes]

According to these researchers, VP adverbs receive a polymorphic type extending over the universe CN (14), while sentence level adverbs are just functions from propositions to propositions (15): ${ }^{14}$

\footnotetext{
${ }^{13}$ It is worth mentioning that subsumptive subtyping, i.e. the traditional notion of subtyping that adopts the subsumption rule (if $A \leq B$, then every object of type $A$ is also of type $B$ ), is inadequate for MTTs in the sense that it would destroy some important metatheoretical properties of MTTs (see, for example, $\S 4$ of [19] for details).

${ }^{14}$ One can see the typing in (14) as a more fine-grained version of Montague's VPadverb typing $(e \rightarrow t) \rightarrow(e \rightarrow t)$. $A$ here stands for the syntactic subject.
} 
(14) $\Pi A:$ CN. $(A \rightarrow$ Prop $) \rightarrow(A \rightarrow$ Prop $)$

(15) Prop $\rightarrow$ Prop

In order to take care of the veridical inference associated with manner adverbs like on time, the authors propose to use an auxiliary object, which they call $A D V$, in effect a $\Sigma$ type:

(16) $A D V: \Pi A: \mathrm{CN} . \Pi v: A \rightarrow \operatorname{Prop} . \Sigma p: A \rightarrow \operatorname{Prop} . \forall x: A . p(x) \supset v(x)$

The above reads as follows: for any common noun $A$ and any predicate $v$ over $A, A D V(A, v)$ is a pair $(p, m)$ such that for any $x: A$, such that $m(x), p(x)$ implies $v(x)$. Then, adverbs like on time or in general adverbs that give rise to this sort of inference can be defined as the first projection of the previous $\Sigma$ type:

(17) on time $=\lambda A: \mathrm{CN} . \lambda v: A \rightarrow$ Prop. $\pi_{1}(A D V(A, v))$

This line of approach, as the authors show, can effectively take care of the inferences associated with veridical adverbs. ${ }^{15}$ In what follows, I present an account of different types of adverbs, concentrating on predicational adverbs and a number of their properties. The veridical/non-veridical distinction is first discussed, followed by a discussion on various other issues associated with the semantics of adverbs like opacity and rich typing. .

\subsection{Veridical adverbs}

A very basic distinction as regards the inference that the semantics of different adverbs give rise to, concerns veridicality. In simple terms, veridical adverbs are adverbs that entail the sentence formed after omitting the adverb. Veridicality is not particular to sentence or VP adverbs and veridical adverbs can be found in both cases. Predicational adverbs involve adverbs of both kinds. Thus, one finds sentence level predicational adverbs like fortunately and frankly, as well as VP adverbs like intentionally and slowly. ${ }^{16}$ The former function on the level of the sentence while the latter at the level of the VP. The $\Sigma$ type account used in [3] for manner adverbs can be adapted to veridical sentence adverbs. This account involves the use of an auxiliary object, followed by the definition of the adverb as the first projection of this auxiliary object. I consider this to be nonsatisfactory for at least two reasons a) redundancy, i.e. every adverb will need

\footnotetext{
${ }^{15}$ It has to be noted that the inference associated with manner adverbs like the one discussed, has been tried out in the Coq proof-assistant $[3,4]$. There it was shown that using a lexical entry for on_time like the one proposed in (17), one can formally verify/prove that (13) is a valid inference. See [3,4] for more details.

${ }^{16}$ Actually, predicational adverbs are further classified as sentence and verb related adverbs according to [21], with further classifications for each case. See [21] for more details. See also [8] for a slightly different classification.
} 
two typings and b) the definition shown (17) involves an equality relation, i.e. it says roughly that these are the semantics of the specific adjective. However, this is just an aspect of the semantics of these adjectives (veridicality). For this reason, I propose to use a single entry for adverbs while still maintaining the $\Sigma$ type mechanism in order to get the veridicality facts right. Thus, the typing for a sentence level adverb like fortunately will be the following:

(18) Fortunately $: \Sigma f:$ Prop $\rightarrow$ Prop. $\forall$ P:Prop. $f(P) \supset P$

In the case of veridical VP-adverbs like for example slowly, fast, the same line of reasoning can be used. However, in this case as well, one has to modify the account proposed in $[3,4]$ for the reasons discussed above. Thus, the new entry for a VP-adverb like slowly will be the one shown below:

(19) Slowly: $\Sigma f: \forall A: C N .(A \rightarrow$ Prop $) \rightarrow(A \rightarrow$ Prop $) . \forall A: C N . \forall v: A \rightarrow$ Prop, $\forall x: A . f(A, v, x) \supset v(x)$

Notice that given $\Sigma$ types are a form of generalized conjunction one can also pursue a davidsonian kind of analysis in case s/he wants to introduce an explicit event argument in the semantics. To give an example, assume, in the spirit of Davidson, that every sentence involves an event argument, with Event : Type. In this case then an intransitive verb like $W a l k$ will have the following type:

(20) Walk: Human $\rightarrow$ Event $\rightarrow$ Prop

With the presence of an explicit event argument, the typing in (19) will be modified as follows: ${ }^{17}$

(21) Slowly : $\Sigma f: \forall A: C N .(A \rightarrow$ Event $\rightarrow$ Prop $) \rightarrow(A \rightarrow$ Event $\rightarrow$ Prop $)$.

$\forall A: C N . \forall v: A \rightarrow$ Event $\rightarrow$ Prop. $\forall x: A . \forall e:$ Event.f $(A, v, e, x) \supset v(e, x) \wedge$ $S L O W(e)$

Of course, veridicality is one of the aspects of adverbs and a number of other issues should be discussed in order to give a complete account of the semantics of various types of adverbs, like e.g. evaluative or subject-oriented adverbs. It is impossible to discuss all these issues here, but however some proposals pertaining to some of these cases will be discussed later on in this paper.

\subsection{Non-veridical adverbs}

It is clear from the above discussion, that non-veridical adverbs should not involve the $\Sigma$ type treatment as this is presented for veridical adverbs, since this will incorrectly predict veridicality. Let us look at some examples in order to see how cases of non-veridical adverbs can be approached. If one is interested in veridicality only, and does not care about the specific semantics of each adverb in each case, a simple assumption as regards the two typings (sentence and VP-adverbs) suffices to prevent unwanted inferences:

$\overline{{ }^{17} \text { With } S L O W}:$ Event $\rightarrow$ Prop. 
(22) $\Pi A:$ CN. $(A \rightarrow$ Prop $) \rightarrow(A \rightarrow$ Prop $)$

(23) Prop $\rightarrow$ Prop

However, one has to say more at least for cases of adverbs like intentionally or epistemic adverbs like possibly or allegedly. The latter case, i.e. epistemic adverbs, creates opaque contexts for both the subject and the object, while the former, i.e intentionally, only for the object:

(24) Oedipus allegedly married Jocaste

(25) Oedipus intentionally married Jocaste

From (24), it does not follow that:

(26) Oedipus allegedly married Jocaste $\nRightarrow$ the son of Laius allegedly married Jocaste

(27) Oedipus married Jocaste $\nRightarrow$ Oedipus allegedly married his mother

On the other hand, from (25) we have:

(28) Oedipus intentionally married Jocaste $\Rightarrow$ The son of Laius intentionally married Jocaste

(29) Oedipus intentionally married Jocaste $\nRightarrow$ Oedipus intentionally married his mother

In order to make sense of these examples, we have to look at how the modal notions associated with adverbs like intentionally and allegedly should be represented. In the first case, one can assume that the meaning of intentionally can be represented as follows: there is an agent $p$, that intentionally did something $x$, in effect meaning that agent $p$ believes that he did $x$. Now, following [27], we can represent $p$ 's belief context as a number of judgments $x: A$ with $A:$ Prop, this agent has made. In case this agent $p$ is Oedipus, this will be the following:

(30) $\Gamma_{\text {oed }}=x_{1}: A_{1}, \ldots, x_{n}: A_{n}\left(x_{1}, \ldots, x_{n-1}\right)$

From this, one can construct a generalized belief operator $B_{p} A$ standing for agent $\mathrm{p}$ believes $\mathrm{A}$. The construction of this operator, is made out of binding all the variables in $\Gamma_{p}$ :

(31) $B_{p} A=\Pi \Gamma_{p} . A=\Pi x_{1}: A_{1} \ldots \Pi x_{n}: A_{n}\left(x_{1}, \ldots, x_{n-1}\right) . A$

Given our knowledge of the story of Oedipus, he does not know neither that he is the son of Laius nor that his mother is Jocaste. In this sense, the required equality relations shown below are not part of Oedipus' belief context:

(32)Eq(Person, Oed, SoL)

(33)Eq(Person, J, MoO) 
Thus, in this case one can assume something like the following for intentionally:

(34) Intentionally $=\lambda A: C N . \lambda P: A \rightarrow$ Prop. $\forall x: A, B_{x}(P(x))$

The above definition says that given a predicate of type $A \rightarrow$ Prop and for all $x: A$ ( $x$ the subject here), the proposition $P(x)$ is true in x's belief context. Thus, in the case of Oedipus intentionally married Jocaste, we get a paraphrase that Oedipus believes/knows that he married Jocaste. Now, how do we derive the non-opacity of the subject, i.e. cases like the son of Laius is Oedipus $\Rightarrow$ The son of Laius married Jocaste? Notice that in (34), the $x$ is not bound by the belief operator. In this sense, given an equality relation $E q($ Person, Oed, SoL $)$ and given $x=O e d$, substitution of Oed with $S o L$ is possible and thus the interpretation where the son of Laius intentionally married Jocaste arises. What one cannot get (correctly) is that Oedipus believes/knows that the son of Laius married Jocaste, according to fact. So, in this sense, the semantics of the adverb are captured as well as the behaviour with respect to opaque contexts. On the other hand, for adjectives like allegedly, one needs a different treatment given the opacity for both subject and object position. Allegedly can then be taken to mean that a given proposition, say $P$, is true in the belief context of an agent $p$ :Human and $p$ is free in $P$. What one needs in this case is a sentence modifer type like the following:

$$
\text { Allegedly }=\lambda P: \text { Prop. } \exists p: H u m a n, B_{p}(P)
$$

In effect, we can deal with these kinds of adjectives without the need of postulating intensions or assuming possible world semantics. This type of approach has the welcoming result that cases of domain adverbs like the ones shown below can receive a similar treatment:

(36) Botanically, tomato is a fruit

(37) Mathematically, the proposition is not correct

These types of adjectives are not veridical, since the proposition expressed is only relevant for the relevant domain in each case (botanology and mathematics respectively). Thus, tomatoes are only fruits only in the context of botanology. These types of adjectives are sentence level adverbs that can be seen to hold in a specified context (i.e. domain).For example, let us assume a context $\Gamma_{B}$ standing for the context representing the collection of facts pertaining to botanology. In this case, botanically will be defined as follows:

(38) Botanically $=\lambda P:$ Prop. $\Gamma_{B} P$

A similar treatment can be given for (37). 
Remark 1. Note that the approach proposed here, similarly to accounts like [26] and [28], predicts that in cases like (39) the only correct interpretation is with painstakingly taking wide scope over intellibly, a fact following from typing given that the former is a sentence adverb while the latter a VP-adverb:

(39) John painstakingly wrote illegibly.

Further differences in interpretation of manner adverbs like the ones shown below can be accounted assuming that in the second case the quantifier takes scope over the adverb while in the first the opposite happens:

(40) Sam carefully sliced all the bagels.

(41) Sam sliced all the bagels carefully.

One can then link, the minimally different interpretations to be dependent on syntactic positioning. One can actually propose a unit type in the sense of [16], encoding the two different interpretations for the same adverb. Then, one can assume that given syntactic positioning, the right type is chosen for each case. The exact mechanism of how this can be done is left for future research.

Remark 2. A note on typing is in place here. Note that the type of VP adverbs, extends over the universe CN. However, in some cases, e.g. with adverbs like intentionally, this will overgenerate since it will predict that non-human or even inanimate entities can be seen as bearing intentions! Fortunately, there is an easy solution in this case. It suffices to assume that besides the universe of common nouns $\mathrm{CN}$ that collects the names of all common noun denotations into a type, there are smaller common noun universes, which are further subuniverses of the already introduced CN universe. This has been already used by [2] in order to deal with a similar type of problem pertaining to adjectives like skilful. In this case, skilful was taken to extend not over the universe $\mathrm{CN}$ but over a subuniverse $\mathrm{CN}_{H}$, i.e the universe containing the names of the types Human and its subtypes. The relevant introduction rules are shown below:

$$
\overline{\text { Human }: \mathrm{CN}_{H}} \frac{A<\text { Human }}{A: \mathrm{CN}_{H}}
$$

. The new entry with this minimal modification is shown below:

Given the above typing, examples like the one shown below are ruled out:

(43) \# The rock intentionally rolled down the hill.

More work on this issue should be done in order to see how the use of such subuniverses can be a useful tool of MTTs in the study of NL semantics. 


\subsection{Conclusions}

This paper presented the first investigation into the semantics of adverbs from a MTT perspective. It was shown that using an expressive formal language like MTTs can have a number of welcoming results with respect to adverb typing. In particular, the issue of veridicality was discussed and a generalized $\Sigma$ type mechanism was proposed for these types of adverbs. Furthermore, the semantics for a number of other cases of adverbs like epistemic, mental-attitude and domain adverbs were proposed by exploiting the type-theoretic notion of context, a notion free of the hyperintensional problems associated with possible world semantics, in order to provide adequate semantics for these cases.

\section{References}

1. Chatzikyriakidis, S., Luo, Z.: An account of natural language coordination in type theory with coercive subtyping. In: Parmentier, Y., Duchier, D. (eds.) Proc. of Constraint Solving and Language Processing (CSLP12). LNCS 8114. pp. 31-51. Orleans (2012)

2. Chatzikyriakidis, S., Luo, Z.: Adjectives in a modern type-theoretical setting. In: Morrill, G., Nederhof, J. (eds.) Proceedings of Formal Grammar 2013. LNCS 8036. pp. 159-174 (2013)

3. Chatzikyriakidis, S., Luo, Z.: Natural language inference in coq (2013), submitted

4. Chatzikyriakidis, S., Luo, Z.: Natural language reasoning using proof-assistant technology: Rich typing and beyond. In: Proceedings of EACL2014 (2014)

5. Church, A.: A formulation of the simple theory of types. J. Symbolic Logic 5(1) (1940)

6. Cinque, G.: Adverbs and Functional Heads: A Cross-Linguistic Perspective. Oxford University Press (1999)

7. Davidson, D.: Compositionality and coercion in semantics: The semantics of adjective meaning. In: Rescher, N. (ed.) The Logical Form of Action Sentences, pp. 81-95. University of Pittsburgh Press (1967)

8. Ernst, T.: The Syntax of Adjuncts. Cambridge press (2002)

9. Ernst, T.: On the role of semantics in a theory of adverb syntax. Lingua 117(6) (2007)

10. Girard, J.Y.: Une extension de l'interpretation fonctionelle de gödel à l'analyse et son application à l'élimination des coupures dans et la thèorie des types'. Proc. 2nd Scandinavian Logic Symposium. North-Holland (1971)

11. Jackendoff, R.: Semantic Interpretation in Generative Grammar. MIT press (1972)

12. Kamp, H.: Formal semantics of natural language. In: Keenan, E. (ed.) Two theories about adjectives, pp. 123-155. Cambridge University Press (1975)

13. Luo, Z.: Computation and Reasoning: A Type Theory for Computer Science. Oxford Univ Press (1994)

14. Luo, Z.: Coercive subtyping. Journal of Logic and Computation 9(1), 105-130 (1999)

15. Luo, Z.: Type-theoretical semantics with coercive subtyping. Semantics and Linguistic Theory 20 (SALT20), Vancouver 84(2), 28-56 (2010)

16. Luo, Z.: Contextual analysis of word meanings in type-theoretical semantics. In: Logical Aspects of Computational Linguistics (LACL'2011). LNAI 6736. pp. 159174 (2011) 
17. Luo, Z.: Common nouns as types. In: Bechet, D., Dikovsky, A. (eds.) Logical Aspects of Computational Linguistics (LACL'2012). LNCS 7351. pp. 173-185 (2012)

18. Luo, Z.: Formal semantics in modern type theories with coercive subtyping. Linguistics and Philosophy 35(6), 491-513 (2012)

19. Luo, Z., Soloviev, S., Xue, T.: Coercive subtyping: theory and implementation. Information and Computation 223, 18-42 (2012)

20. Luo, Z.: Adjectives and adverbs in type-theoretical semantics. Notes (2011)

21. Maienborn, C., Schafer, M.: Adverbs and adverbials. In: Maienborn, C., von Heusinger, K., Portner, P. (eds.) Semantics. An international handbook of natural language meaning, pp. 1390-1420. Mouton: De Gruyter (2011)

22. Martin-Löf, P.: An intuitionistic theory of types: predicative part. In: H.Rose, J.C.Shepherdson (eds.) Logic Colloquium'73 (1975)

23. Martin-Löf, P.: Intuitionistic Type Theory. Bibliopolis (1984)

24. Montague, R.: English as a formal language. In: Visentini, B. (ed.) Linguaggi nella Societ e nella. Milan: Edizioni di Comunit,l (1970)

25. Montague, R.: Formal Philosophy. Yale University Press (1974)

26. Parsons, T.: Some problems concerning the logic of grammatical modifiers. In: Davidson, D., Harman, G. (eds.) Semantics of Natural Language., pp. 127-141. Dordrecht:Reidel (1972)

27. Ranta, A.: Type-Theoretical Grammar. Oxford University Press (1994)

28. Thomason, R., R.C., S.: A semantic theory of adverbs. Linguistic Inquiry 4(2), 195-220 (1973) 